\title{
COMPOSIÇÃO FLORÍSTICA DE DOIS TRECHOS EM DIFERENTES ETAPAS SERAIS DE UMA FLORESTA ESTACIONAL SEMIDECIDUAL EM VIÇOSA, MINAS GERAIS ${ }^{1}$
}

\author{
Rogério Ferreira Ribas ${ }^{2}$, João Augusto Alves Meira Neto ${ }^{3}$, Alexandre Francisco da Silva ${ }^{3}$ e \\ Agostinho Lopes de Souza ${ }^{4}$
}

\begin{abstract}
RESUMO - O presente estudo foi desenvolvido em dois trechos de floresta semidecídua, com diferentes estádios de sucessão secundária, localizada no município de Viçosa-MG, objetivando verificar variações qualitativas na composição florística. Foram demarcadas, em cada trecho, dez parcelas de 10 x $20 \mathrm{~m}$, nas quais foram inventariados todos os indivíduos lenhosos com circunferência à altura de $130 \mathrm{~cm}$ do solo maior ou igual a $5 \mathrm{~cm}$. A similaridade florística entre as parcelas foi avaliada por meio de análise de agrupamentos, utilizando o índice de Sørensen. Foram amostradas 67 espécies no trecho com 15 anos e 69 no trecho com 30 anos. Dentre as espécies exclusivas, 38 foram para o trecho com 15 anos e 40 para o trecho com 30 anos. As famílias Annonaceae, Leguminosae Caesalpinioideae e Sapindaceae, com cinco, quatro e quatro espécies cada, respectivamente, e o gênero Nectandra, com duas espécies, foram os taxa mais bem representados no trecho com 15 anos. Por outro lado, as famílias Flacourtiaceae, Meliaceae e Myrtaceae, com quatro, três e quatro espécies, respectivamente, e o gênero Ocotea, com três espécies, foram mais bem representados no trecho com 30 anos. A similaridade florística entre parcelas de um mesmo trecho foi considerada alta, tendo sido relacionada à proximidade espacial, o que implica históricos de perturbação e regeneração semelhantes, resultando, conseqüentemente, em composições florísticas mais similares e mesmo estádio de sucessão secundária.
\end{abstract}

Palavras-chave: Floresta estacional semidecidual, grupos funcionais, composição florística e sucessão secundária.

\section{FLORISTIC COMPOSITION OF TWO SITES IN DIFFERENT SERAL STAGES OF SEMIDECIDUOUS SEASONAL MONTANE FOREST IN VIÇOSA, MINAS GERAIS}

\begin{abstract}
This study was carried out in two sites of a semideciduous forest, under different stages of secondary succession in Viçosa, MG to verify qualitative variations in floristic composition. In each site, ten plots of $10 \mathrm{mx}$ $20 \mathrm{~m}$ were delimited, with all woody individuals with circumference at $130 \mathrm{~cm}$ height from the soil larger than or equal to $5 \mathrm{~cm}$ being listed. Floristic similarity among parcels was evaluated through clustering analysis, using the Sorensen index. Sixty-seven species were sampled in the 15-year-old area, and 69 species in the 30-year-old area. Among the exclusive species, 38 were placed in the younger site and 40 in the older. The families Annonaceae, Leguminosae Caesalpinioideae and Sapindaceae, with five, four and four species, respectively, and the genus Nectandra, with two species, were better represented in the 15-year-old site. On the other hand, the families Flacourtiaceae, Meliaceae and Myrtaceae, with four, three and four species, respectively, and the genus Ocotea, with three species, were better represented in the 30-year-old site. Floristic similarity among plots from the same area was considered high, being related to spatial proximity, which reflects similar bakgrounds of disturbance and regeneration, consequently resulting in similar floristic compositions and secondary succession stage.
\end{abstract}

Key words: Tropical semideciduous forest, functional groups, floristic composition, secondary succession.

1 Recebido para publicação em 26.11.2001.

Aceito para publicação em 4.11.2003.

Parte da tese do primeiro autor, apresentada à Universidade Federal de Viçosa. Trabalho realizado com o auxílio da CAPES e FAPEMIG.

2 Biólogo, Mestre em Botânica, <ribascr@insecta.ufv.br>; ${ }^{3}$ Dep. de Biologia Vegetal da Universidade Federal de Viçosa UFV; ${ }^{4}$ Dep. de Engenharia Florestal - UFV, 36570-000 Viçosa-MG. 


\section{INTRODUÇÃO}

Nas últimas décadas, a área ocupada por florestas em regeneração no Estado de Minas Gerais vem se expandindo rapidamente, em decorrência da crescente alteração e retirada de florestas primárias para extração seletiva de madeira e, na sua maior parte, para formação de pastagens ou outras culturas, com posterior abandono. Segundo a FUNDAÇÃO CENTRO TECNOLÓGICO DE MINAS GERAIS-CETEC (1983), a floresta secundária substituiu, praticamente, todas as florestas primárias existentes no Estado, restando apenas pequenas manchas remanescentes, que correspondem a menos de $2 \%$ do território mineiro.

As Florestas Estacionais Semideciduais da Zona da Mata de Minas Gerais, em particular, foram severamente modificadas pelo ciclo do café e pela pecuária extensiva e, mais recentemente, pelo plantio da cana-de-açúcar (Valverde, 1958; Meira-Neto \& Silva, 1995). Como resultado, houve a formação de paisagens que consistem de remanescentes esparsos de floresta primária, dominados por fragmentos de floresta secundária de várias idades, pastagens e campos de cultivo.

Devido à sua dominância na Zona da Mata de Minas Gerais, as florestas semideciduais em regeneração representam um recurso natural de relevância para a exploração econômica, bem como para a subsistência das populações locais. Além disto, as florestas semideciduais em regeneração são também um importante laboratório natural, capaz de gerar informações indispensáveis ao entendimento da dinâmica sucessional dessas comunidades florestais. Neste sentindo, o inventário da flora, aliado ao estudo de ecologia de comunidades vegetais, é considerado fundamental e prévio para embasar quaisquer atividades de uso, manejo, recuperação e conservação da floresta semidecídua.

Diante desta constatação e também da ausência de estudos detalhados de diferentes fases sucessionais das florestas secundárias na Zona da Mata de Minas Gerais, foi realizado o estudo da composição florística de dois trechos de um fragmento de Floresta Estacional Semidecidual Montana, com diferentes estádios de sucessão secundária, no município de Viçosa, Minas Gerais.

\section{MATERIAL E MÉTODOS}

\section{1. Área de Estudo}

A área estudada, com 5 ha, está em uma propriedade particular, o sítio Palmital, localizado entre as coordenadas $20^{\circ} 48^{\prime} 35^{\prime \prime}$ a $20^{\circ} 48^{\prime} 50^{\prime \prime} \mathrm{S}$ e $42^{\circ} 50^{\prime} 50^{\prime \prime}$ a $42^{\circ} 51^{\prime} 10^{\prime \prime} \mathrm{W}$, próximo à região urbana do município de Viçosa. Está inserida em um prolongamento da Serra da Mantiqueira, em uma divisão geopolítica conhecida como Zona da Mata de Minas Gerais, entre altitudes que variam de 200 a 900 m (Valverde, 1958). A topografia da região é acidentada, com vales estreitos e úmidos, e o relevo varia de fortemente ondulado a montanhoso (MariscalFlores, 1993).

Conforme classificação climática de Köppen, o clima regional é do tipo Cwb, mesotérmico úmido com verões chuvosos e invernos secos (Vianello \& Alves, 1991). A precipitação média anual é de $1.221,4 \mathrm{~mm}$ (DEPARTAMENTO NACIONAL DE METEREOLOGIA, 1992), devendo-se ressaltar que o balanço hídrico, segundo Thornthwaite \& Mather (1955), citados por Golfari (1975), mostra a existência de um período com déficit hídrico de maio a setembro e excedente de dezembro a março. Dados climatológicos indicam umidade relativa do ar em torno de $80 \%$ e temperatura média anual variando de $26,1{ }^{\circ} \mathrm{C}$ para a máxima e $14,0{ }^{\circ} \mathrm{C}$ para a mínima (Castro et al., 1973). De acordo com Veloso et al. (1991), a vegetação desta região é classificada como Floresta Estacional Semidecidual Montana, caracterizada pela mistura de espécies caducifólias e perenifólias, estabelecidas acima de $500 \mathrm{~m}$ de altitude.

Fisiograficamente, a mata do sítio do Palmital situase no alto até o meio de uma encosta, apresentando declividade média de $45 \%$. Segundo moradores locais, o fragmento de mata em questão passou por desmatamento completo para implantação de cultura do café, abandonada há pelo menos 30 anos. Em meados da década de 80, parte da vegetação em regeneração natural, estabelecida na meia encosta, foi submetida a corte raso e queima, restando apenas algumas árvores esparsas. Após esse período a área entrou em progressivo processo de regeneração natural. Atualmente, a vegetação apresenta-se composta por dois trechos de floresta secundária em diferentes idades de regeneração.

\subsection{Florística}

Para o estudo da composição florística foram instaladas 20 parcelas de $10 \times 20 \mathrm{~m}$, distribuídas, sistematicamente, em dois blocos de dez parcelas. Os blocos foram alocados de forma a amostrar duas áreas diferentes da mata: um trecho de floresta com 15 anos, situado na meia-encosta, e outro localizado na altaencosta, com idade aproximada de 30 anos. Nas duas 
áreas foram incluídas na amostragem todas as plantas lenhosas eretas, cuja circunferência do caule a 1,3 m de altura do solo fosse igual ou maior que $5 \mathrm{~cm}$ (CAP), equivalente a um diâmetro de $1,59 \mathrm{~cm}$. A amostragem foi realizada no período de março a dezembro de 1999.

O sistema de classificação adotado para elaboração da lista de espécies foi proposto por Cronquist (1988). Os nomes das espécies foram confirmados e atualizados pelo software do índice de espécies do ROYAL BOTANIC GARDENS OF KEW (1993) ou por meio de literatura especializada, cujas informações são mais atualizadas que as desse programa. $\mathrm{O}$ material botânico coletado em estado reprodutivo está depositado no Herbário VIC do Departamento de Biologia Vegetal da Universidade Federal de Viçosa.

A similaridade florística entre as parcelas amostradas foi verificada por meio de uma análise de agrupamentos, utilizando o índice de similaridade de Sørensen (Brower \& Zar, 1984). Para interpretar a similaridade florística entre cada uma das unidades amostrais, utilizouse o método de médias não-ponderadas (UPGMA), que resulta em um dendrograma de classificação hierárquica aglomerativa, baseada na distância média mínima entre os grupos de parcelas e que expressa graficamente as relações de similaridade entre essas unidades (Sneath \& Sokal, 1973). Para efeito de comparação, empregaram-se ainda os métodos de ligação simples e de ligação completa, que reuniram os objetos (unidades de observação) de um grupo pela maior ou menor semelhança, respectivamente (Sneath \& Sokal, 1973). Os dendrogramas foram produzidos por meio do programa FITOPAC 1 (Shepherd, 1996).

\section{RESULTADOS E DISCUSSÃO}

Como resultado do levantamento florístico verificou-se que 67 espécies foram amostradas no trecho com 15 anos e 69 no trecho com 30 anos. Embora pouco expressivo, esse dado indica um aumento da riqueza de espécies durante o processo de regeneração secundária na área estudada. Essa tendência é comumente citada para outras florestas tropicais do Brasil (Leitão-Filho, 1993; Tabarelli \& Mantovani, 1999). Nesses estudos, durante a regeneração foram observadas poucas espécies nas florestas em estádios iniciais da sucessão secundária e riquezas maiores nas florestas mais desenvolvidas, onde costumam coexistir espécies de diversas fases da sucessão.

Dentre as espécies exclusivas, 38 pertencem ao trecho com 15 anos e 40 ao trecho com 30 anos. Várias espécies estiveram presentes em mais de uma área, de forma que nos dois trechos foi relacionado um total de 107 espécies, pertencentes a 81 gêneros, representando 35 famílias botânicas (Quadro1).

Quadro 1 - Lista florística das espécies amostradas nos trechos com 15 e 30 anos da mata do sítio do Palmital, município de Viçosa-MG, apresentada em ordem alfabética de famílias, gêneros e espécies

Table 1 - Floristic list of species sampled in 15- and 30-year-old sites in Sítio do Palmital, Viçosa, MG, presented in alphabetical order of families, genera and species

\begin{tabular}{|c|c|c|c|}
\hline Família/Espécie & Nome Comum & $\begin{array}{c}15 \\
\text { anos }\end{array}$ & $\begin{array}{c}30 \\
\text { anos }\end{array}$ \\
\hline \multicolumn{4}{|l|}{ ANACARDIACEAE } \\
\hline Tapirira guianensis Aubl. & fruta-de-pombo & $\mathrm{x}$ & $\mathrm{x}$ \\
\hline \multicolumn{4}{|l|}{ ANNONACEAE } \\
\hline Annona cacans Warm. & araticum-cagão & $\mathrm{x}$ & \\
\hline Guatteria villosissima A.St.-Hil.' & araticum-peludo & $\mathrm{x}$ & \\
\hline Rollinia laurifolia Schltdl. & araticum-bravo & $\mathrm{x}$ & $\mathrm{x}$ \\
\hline Rollinia sericea (R.E.Fr.) R.E.Fr. & araticum-mirim & $\mathrm{x}$ & \\
\hline Xylopia sericea A.St.-Hil. & pimenteira & $\mathrm{x}$ & $\mathrm{x}$ \\
\hline \multicolumn{4}{|l|}{ APOCYNACEAE } \\
\hline Himatanthus phagedaenicus (Mart.) Woodson & sucuúba & & $\mathrm{x}$ \\
\hline Peschiera laeta Miers & & $\mathrm{x}$ & \\
\hline
\end{tabular}


Quadro 1, cont.

Table 1, cont.

\begin{tabular}{|c|c|c|c|}
\hline Família/Espécie & Nome Comum & $\begin{array}{c}15 \\
\text { anos }\end{array}$ & $\begin{array}{c}30 \\
\text { anos }\end{array}$ \\
\hline \multicolumn{4}{|l|}{ ARECACEAE } \\
\hline Syagrus romanzoffiana (Cham.) Glassman & jerivá & $\mathrm{x}$ & \\
\hline \multicolumn{4}{|l|}{ ASTERACEAE } \\
\hline Baccharis sp. & & $\mathrm{x}$ & \\
\hline Vernonia diffusa Less. & vassourão-preto & $\mathrm{x}$ & $\mathrm{x}$ \\
\hline \multicolumn{4}{|l|}{ BIGNONIACEAE } \\
\hline Jacaranda macrantha Cham. & caroba & & $\mathrm{x}$ \\
\hline Sparattosperma leucanthum K. Schum. & cinco-folhas & $\mathrm{x}$ & $\mathrm{x}$ \\
\hline \multicolumn{4}{|l|}{ BORAGINACEAE } \\
\hline Cordia ecalyculata Vell. & porangaba & $\mathrm{x}$ & \\
\hline Cordia sellowiana Cham. & chá-de-bugre & & $\mathrm{x}$ \\
\hline Cordia sp. & & $\mathrm{x}$ & \\
\hline \multicolumn{4}{|l|}{ CECROPIACEAE } \\
\hline Cecropia glaziovi Snethl. & embaúba-vermelha & $\mathrm{x}$ & $\mathrm{x}$ \\
\hline Cecropia hololeuca Miq. & embaúba-branca & $\mathrm{x}$ & $\mathrm{x}$ \\
\hline \multicolumn{4}{|l|}{ CHRYSOBALANACEAE } \\
\hline Hirtella hebeclada Moric. ex A. DC. & azeitona-da-mata & & $\mathrm{x}$ \\
\hline Hirtella selloana Hook. f. & & & $\mathrm{x}$ \\
\hline \multicolumn{4}{|l|}{ CUNONIACEAE } \\
\hline Lamanonia ternata Vell. & cedrilho & & $\mathrm{x}$ \\
\hline \multicolumn{4}{|l|}{ ELAEOCARPACEAE } \\
\hline Sloanea monosperma Vell. & sapopeba & & $\mathrm{x}$ \\
\hline \multicolumn{4}{|l|}{ ERYTHROXYLACEAE } \\
\hline Erythroxylum pelleterianum A.St.-Hil.. & cocão & $\mathrm{x}$ & \\
\hline \multicolumn{4}{|l|}{ EUPHORBIACEAE } \\
\hline Alchornea triplinervia Müll. Arg. & irucurana & & $\mathrm{x}$ \\
\hline Hieronyma alchorneoides Allemão & licurana & & $\mathrm{x}$ \\
\hline Manihot dulcis Baill. & maniçoba & $x$ & \\
\hline Maprounea guianensis Aubl. & carambola-da-mata & $\mathrm{x}$ & $\mathrm{x}$ \\
\hline Sapium glandulatum (Vell.) Pax & leiteiro & $\mathrm{x}$ & \\
\hline \multicolumn{4}{|l|}{ FLACOURTIACEAE } \\
\hline Carpotroche brasiliensis Endl. & canudo-de-pito & $\mathrm{x}$ & $\mathrm{x}$ \\
\hline Casearia arborea Urb. & & & $\mathrm{x}$ \\
\hline Casearia decandra Jacq. & café-do-mato & & $\mathrm{x}$ \\
\hline Casearia ulmifolia Cambess." & cafezinho & & $x$ \\
\hline Xylosma prockia (Turcz.) Turcz. & espinho-de-judeu & $\mathrm{x}$ & \\
\hline \multicolumn{4}{|l|}{ GUTTIFERAE } \\
\hline Kielmeyera sp. & & & $\mathrm{x}$ \\
\hline Rheedia gardneriana Planch. \& Triana & bacupari & & $\mathrm{x}$ \\
\hline Vismia martiana Rechb. f. & ruão & & $\mathrm{x}$ \\
\hline \multicolumn{4}{|l|}{ LABIATAE } \\
\hline Hyptis cana Pohl ex Benth.' & hortelã-do-campo & $\mathrm{x}$ & $\mathrm{x}$ \\
\hline \multicolumn{4}{|l|}{ LACISTEMACEAE } \\
\hline Lacistema pubescens Mart. & & $\mathrm{x}$ & $\mathrm{x}$ \\
\hline
\end{tabular}

R. Árvore, Viçosa-MG, v.27, n.6, p.821-830, 2003 
Quadro 1, cont.

Table 1, cont.

\begin{tabular}{|c|c|c|c|}
\hline Família/Espécie & Nome Comum & $\begin{array}{c}15 \\
\text { anos }\end{array}$ & $\begin{array}{c}30 \\
\text { anos }\end{array}$ \\
\hline \multicolumn{4}{|l|}{ LAURACEAE } \\
\hline Nectandra lanceolata Nees \& Mart. ex Nees & & $\mathrm{x}$ & \\
\hline Nectandra rigida Nees & canela-amarela & $\mathrm{x}$ & $\mathrm{x}$ \\
\hline Ocotea corymbosa $\mathrm{Mez}$ & canela-fedida & & $\mathrm{x}$ \\
\hline Ocotea dispersa $\mathrm{Mez}$ & canelinha & & $\mathrm{x}$ \\
\hline Ocotea odorifera (Vell.) Rohwer & canela-sassafrás & $\mathrm{x}$ & $\mathrm{x}$ \\
\hline Lauraceae sp. & & & $\mathrm{x}$ \\
\hline \multicolumn{4}{|l|}{ LEGUMINOSAE CAESALPINIOIDEAE } \\
\hline Apuleia leiocarpa J.F. Macbr. & garapa & $\mathrm{x}$ & $\mathrm{x}$ \\
\hline Peltophorum dubium Taub. & farinha-seca & $\mathrm{x}$ & \\
\hline Senna macranthera (DC. ex Collad.) Irwin \& Barneby & aleluia & $\mathrm{x}$ & \\
\hline Swartzia sp. & & $x$ & \\
\hline \multicolumn{4}{|l|}{ LEGUMINOSAE MIMOSOIDEAE } \\
\hline Anadenanthera colubrina (Vell.) Brenan & angico-branco & & $\mathrm{x}$ \\
\hline Enterolobium contortisiliquum (Vell.) Morong & tamboril & $\mathrm{x}$ & \\
\hline Inga cylindrica (Vell.) Mart & ingá-mirim & & $\mathrm{x}$ \\
\hline Inga vera Willd. & ingá & & $\mathrm{x}$ \\
\hline Piptadenia gonoacantha J.F. Macbr. & jacaré & $\mathrm{x}$ & $\mathrm{x}$ \\
\hline Plathymenia foliolosa Benth. & vinhático & $\mathrm{x}$ & \\
\hline Pseudopiptadenia contorta (DC.) G.P. Lewis \& M.P. Lima & & & $\mathrm{x}$ \\
\hline Stryphnodendron guianense Benth. & & & $\mathrm{x}$ \\
\hline \multicolumn{4}{|l|}{ LEGUMINOSAE PAPILIONOIDEAE } \\
\hline Andira fraxinifolia Benth. & angelim & & $\mathrm{x}$ \\
\hline Dalbergia nigra Allemão ex Benth. & jacaranda-caviúna & $x$ & $\mathrm{x}$ \\
\hline Dalbergia variabilis Vogel & & $\mathrm{x}$ & \\
\hline Machaerium brasiliense Vogel & canela-do-brejo & $\mathrm{x}$ & $\mathrm{x}$ \\
\hline Machaerium sp. & & $\mathrm{x}$ & \\
\hline Platymiscium pubescens Micheli & & $\mathrm{x}$ & \\
\hline \multicolumn{4}{|l|}{ MELASTOMATACEAE } \\
\hline Miconia cubatanensis Hoehne & & & $\mathrm{x}$ \\
\hline Miconia sellowiana Naudin & jacatirão & & $\mathrm{x}$ \\
\hline Miconia urophylla DC. & & & $\mathrm{x}$ \\
\hline \multicolumn{4}{|l|}{ MELIACEAE } \\
\hline Cabralea canjerana (Vell.) Mart. & cangerana & & $\mathrm{x}$ \\
\hline Guarea kunthiana A.Juss. & andirobarana & & $\mathrm{x}$ \\
\hline Trichilia lepidota Mart. & & $\mathrm{x}$ & $\mathrm{x}$ \\
\hline \multicolumn{4}{|l|}{ MONIMIACEAE } \\
\hline Siparuna guianensis Aubl.' & folha-santa & $\mathrm{x}$ & $\mathrm{x}$ \\
\hline Siparuna reginae A.DC. & & $x$ & $\mathrm{x}$ \\
\hline
\end{tabular}


Quadro 1, cont.

Table 1, cont.

\begin{tabular}{|c|c|c|c|}
\hline Família/Espécie & Nome Comum & $\begin{array}{c}15 \\
\text { anos }\end{array}$ & $\begin{array}{c}30 \\
\text { anos }\end{array}$ \\
\hline \multicolumn{4}{|l|}{ MORACEAE } \\
\hline Brosimum glaziovii Taub. & & $\mathrm{x}$ & $\mathrm{x}$ \\
\hline Ficus guaranitica Chodat & figueira-branca & $\mathrm{x}$ & $\mathrm{x}$ \\
\hline Maclura tinctoria D.Don ex Steud. & amoreira & $\mathrm{x}$ & $\mathrm{x}$ \\
\hline Sorocea bomplandii (Baill.) Bürger, Lanj. \& Boer & folha-de-serra & & $\mathrm{x}$ \\
\hline \multicolumn{4}{|l|}{ MYRTACEAE } \\
\hline Eugenia leptoclada Berg & & & $\mathrm{x}$ \\
\hline Eugenia sp. & & & $\mathrm{x}$ \\
\hline Myrcia fallax DC. & & & $x$ \\
\hline Myrcia sp. & & & $\mathrm{x}$ \\
\hline Psidium cattleyanum Sabine & araçá-do-mato & $\mathrm{x}$ & \\
\hline \multicolumn{4}{|l|}{ NYCTAGINACEAE } \\
\hline Guapira opposita (Vell.) Reitz & maria-mole & $\mathrm{x}$ & \\
\hline \multicolumn{4}{|l|}{ RUBIACEAE } \\
\hline Alibertia sp. & & & $\mathrm{x}$ \\
\hline Amaioua guianensis Aubl. & carvoeiro & & $\mathrm{x}$ \\
\hline Bathysa nicholsonii K. Schum. & & $\mathrm{x}$ & $\mathrm{x}$ \\
\hline Guettarda viburnoides Cham. \& Schltdl. & angélica & $\mathrm{x}$ & $\mathrm{x}$ \\
\hline Randia armata DC. & limorana & $\mathrm{x}$ & \\
\hline Rubiaceae sp. & & & $\mathrm{x}$ \\
\hline \multicolumn{4}{|l|}{ RUTACEAE } \\
\hline Dictyoloma vandellianum A.Juss. & sabugueiro-do-mato & $\mathrm{x}$ & $\mathrm{x}$ \\
\hline Zanthoxylum rhoifolium Lam. & maminha-de-porca & $\mathrm{x}$ & $\mathrm{x}$ \\
\hline \multicolumn{4}{|l|}{ SAPINDACEAE } \\
\hline Allophylus edulis (A.St.-Hil..) Radlk. ex Warm. & vacunzeiro & $\mathrm{x}$ & \\
\hline Allophylus petiolulatus Radlk. ex W.Muell. & casca-solta & & $\mathrm{x}$ \\
\hline Allophylus sericeus Radlk. & três-folhas & $\mathrm{x}$ & \\
\hline Cupania vernalis Cambess. & pau-de-cantil & $\mathrm{x}$ & \\
\hline Matayba elaeagnoides Radlk. & camboatá & $\mathrm{x}$ & $\mathrm{x}$ \\
\hline \multicolumn{4}{|l|}{ SAPOTACEAE } \\
\hline Chrysophyllum gonocarpum (Mart. \& Eckl.) Engl. & guatambu-sapo & & $\mathrm{x}$ \\
\hline \multicolumn{4}{|l|}{ SOLANACEAE } \\
\hline Cestrum sendtnerianum Mart. ex Sendtn. & coerana & $\mathrm{x}$ & \\
\hline Solanum cernuum Vell. & & $\mathrm{x}$ & \\
\hline Solanum leucodendron Sendtn. & adrago & $\mathrm{x}$ & \\
\hline Solanum mauritianum Scop. & & $\mathrm{x}$ & \\
\hline Solanum robustum H.Wendl. & & $\mathrm{x}$ & \\
\hline Solanum swartzianum Roem. \& Schult. & & $\mathrm{X}$ & \\
\hline Solanum sp. & & $\mathrm{x}$ & \\
\hline \multicolumn{4}{|l|}{ TILIACEAE } \\
\hline Luehea grandiflora Mart. & açoita-cavalo & $\mathrm{x}$ & \\
\hline Triumfetta semitriloba Jacq. & carrapichão & $\mathrm{x}$ & \\
\hline \multicolumn{4}{|l|}{ ULMACEAE } \\
\hline Trema micrantha Blume & & $\mathrm{x}$ & \\
\hline \multicolumn{4}{|l|}{ VERBENACEAE } \\
\hline Aegiphila sellowiana Cham. & capoeirão & $\mathrm{X}$ & \\
\hline Vitex sellowiana Cham. & tarumã & $\mathrm{X}$ & $\mathrm{X}$ \\
\hline
\end{tabular}


O número total de espécies (107) obtido neste estudo pode ser considerado expressivo em relação à maioria dos trabalhos realizados em florestas estacionais semideciduais na Zona da Mata de Minas Gerais. Valores inferiores foram citados por Mariscal-Flores (1993), 91, por Soares-Júnior (2000), 83, e por Silva et al. (2000), 91, todos em Viçosa; por Almeida \& Souza (1997), 78, em Juiz de Fora; e por Meira-Neto et al. (1997), 89, em Ponte Nova. Valor idêntico foi encontrado por Senra (2000), 107, em Viçosa. Valores superiores foram obtidos por Meira-Neto (1997), 154, e por Marangon (1999), 182, ambos em Viçosa. Contudo, é importante ressaltar que os diferentes critérios de inclusão de indivíduos e as diferenças de esforço amostral despendido, nos levantamentos citados, limitam as comparações e, portanto, todas estas devem ser feitas com muito critério. Além disto, ressalvando-se essas diferenças metodológicas, estudos realizados em florestas tropicais indicam que o estádio sucessional e o histórico de perturbação da floresta podem influenciar o número de espécies encontrado na comunidade (Leitão-Filho, 1993; Tabarelli \& Mantovani, 1999; Werneck et al., 2000).

Com referência às famílias que apresentaram maior número de espécies, por ordem decrescente, estão: Leguminosae Mimosoideae com oito, Solanaceae com sete, Euphorbiaceae, Leguminosae Papilionoideae, Lauraceae e Rubiaceae com seis e, Annonaceae, Flacourtiaceae, Myrtaceae e Sapindaceae com cinco. Várias dessas famílias aparecem, com destaque, dentre as mais representativas em número de espécies, na maioria dos estudos realizados em florestas semidecíduas no Estado de Minas Gerais (Oliveira-Filho \& Machado, 1993; Oliveira-Filho et al., 1994; Paula, 1999; Carvalho et al., 2000; Soares-Júnior, 2000 Werneck et al., 2000). A família de maior riqueza específica neste estudo, Leguminosae Mimosoideae, também ocupou posição expressiva em outros fragmentos secundários de Floresta Estacional Semidecidual Montana na região de Viçosa-MG (Meira-Neto, 1997; Almeida-Júnior, 1999; Senra, 2000). Das famílias amostradas, Arecaceae, Erythroxylaceae, Nyctaginaceae, Solanaceae, Tiliaceae e Ulmaceae ocorreram exclusivamente no trecho com 15 anos. Por outro lado, Chrysobalanaceae, Cunoniaceae, Elaeocarpaceae, Guttiferae, Melastomataceae e Sapotaceae foram de ocorrência exclusiva do trecho com 30 anos. As famílias Annonaceae, Leguminosae Caesalpinioideae e Sapindaceae, com cinco, quatro e quatro espécies cada, respectivamente, e o gênero Nectandra, com duas espécies, foram mais bem representados no trecho com 15 anos. Por outro lado, as famílias Flacourtiaceae, Meliaceae e Myrtaceae, com quatro, três e quatro espécies, respectivamente, e o gênero Ocotea, com três espécies, foram mais bem representados no trecho com 30 anos (Quadro 1). Entretanto, deve-se considerar que outros fatores ambientais não-estudados podem exercer influência na alteração da composição, embora a proximidade dos trechos amostrados minimize esse efeito.

Com relação à análise de agrupamentos, verificouse que os mais altos índices de similaridade foram encontrados entre parcelas vizinhas de um mesmo trecho. Em contrapartida, menores semelhanças foram obtidas entre parcelas alocadas em trechos distintos do fragmento. A maior similaridade florística $(70,6 \%)$ foi encontrada entre as parcelas 14 e 15 e a menor similaridade $(9,09 \%)$ ocorreu entre as parcelas 2 e 19. A explicação das maiores semelhanças apresentadas entre as parcelas de um mesmo trecho está relacionada à proximidade espacial, o que implica históricos de perturbação e regeneração semelhantes, resultando, conseqüentemente, em composições florísticas mais similares e mesmo estádio de sucessão secundária.

As relações florísticas, visualizadas por meio do dendrograma gerado pela análise de agrupamentos (UPGMA) a partir dos índices de similaridades de Sørensen, estão representadas na Figura 1 (a). O dendrograma mostra, graficamente, que foram formados dois grupos de amostras. O primeiro, com similaridade em nível de $48,8 \%$, foi estabelecido para as parcelas alocadas no trecho com 30 anos (de 11 a 20), enquanto o segundo, em nível de similaridade de 46,2\%, agrupou as parcelas alocadas no trecho com 15 anos (de 1 a 10). Comparando os dendrogramas obtidos pelos métodos de médias nãoponderadas, ligação simples (Figura 1b) e ligação completa (Figura 1c), constata-se que a formação desses grupos de amostras foi comum aos três métodos.

As diferenças florísticas entre os trechos de floresta, encontradas neste estudo, fazem com que o trecho de 15 anos tenha todas suas parcelas formando um grupo inequívoco, divergindo de todas as parcelas do trecho com 30 anos, que formam outro grupo distinto na análise de agrupamentos. Esses grupos de similaridade assim estabelecidos estão relacionados, provavelmente, às particularidades ecológicas de cada trecho. Diferentes históricos de perturbação e regeneração, além de variações de fatores ambientais, como a qualidade e quantidade de luz e a disponibilidade de água e nutrientes do solo, resultaram, no decorrer do processo de regeneração 
(a) Hesa de Gespa postes

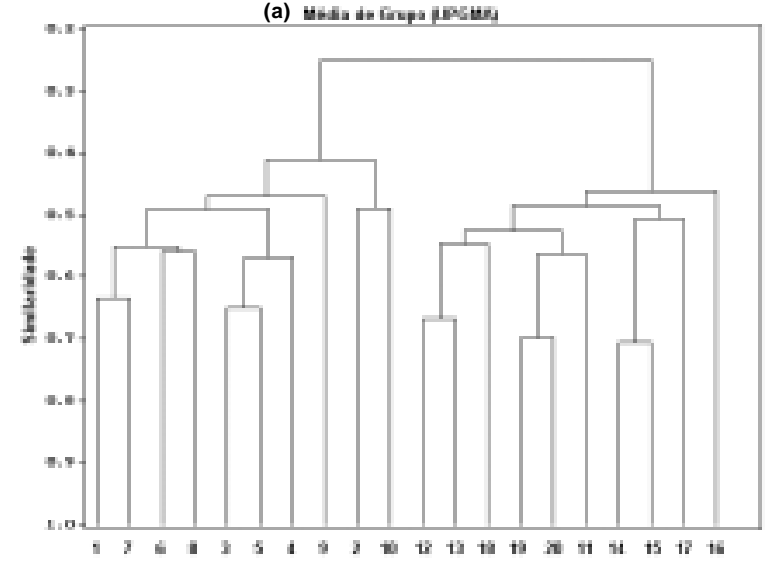

(b) Iasats 5 tarte

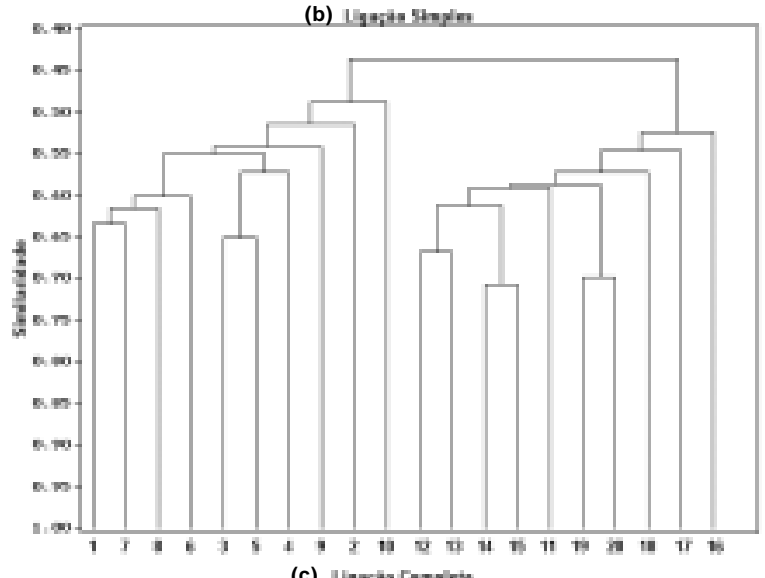

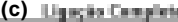

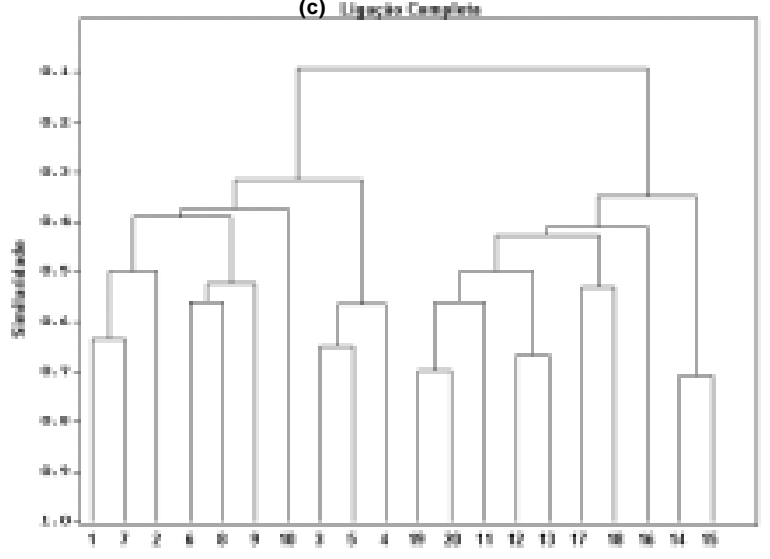

Figura 1 - Dendrogramas obtidos pelos métodos UPGMA (a), ligação simples (b) e ligação completa (c), com base no índice de Sørensen, para as parcelas do sítio do Palmital (Viçosa-MG). As parcelas de 1 a 10 referem-se ao trecho com 15 anos e as parcelas de 11 a 20 referemse ao trecho com 30 anos.

Figure 1 - Dendograms obtained by the methods UPGMA (a), single linkage (b) and complete linkage (c), using Soresen index, for the parcels in the Sitio do Palmital (Viçosa, Mg). Parcels 1 up to 10 refer to the 15-year-old site, and parcels 11 to 20 refer to the 30-year-old site. natural, em manchas com diferentes composições florísticas, contribuindo para a manutenção ou o aumento das diferenças florísticas entre os trechos da floresta.

\section{CONCLUSÕES}

Embora o trecho de floresta com 15 anos tenha apresentado número de espécies próximo ao do trecho com 30 anos (67 e 69 espécies, respectivamente), a tendência é que haja aumento da riqueza de espécies em função do avanço do processo sucessional.

Durante o processo de sucessão secundária desse fragmento florestal, de 15 para 30 anos, Erythroxylaceae, Nyctaginaceae, Solanaceae, Tiliaceae, Ulmaceae, Annonaceae, Leguminosae Caesalpinioideae, Sapindaceae e o gênero Nectandra tendem a ser substituídos por Chrysobalanaceae, Cunoniaceae, Elaeocarpaceae, Guttiferae, Melastomataceae, Sapotaceae, Flacourtiaceae, Meliaceae, Myrtaceae e o gênero Ocotea. Entretanto, deve-se considerar que outros fatores não-estudados podem exercer influência na alteração da composição, embora a proximidade dos trechos amostrados minimize esse efeito.

A similaridade florística entre parcelas de um mesmo trecho foi considerada alta, estando relacionada a históricos de perturbação e regeneração semelhantes, o que implica o mesmo estádio de sucessão secundária, resultando, conseqüentemente, em composições florísticas mais similares.

De modo geral, pode-se dizer que o processo de sucessão secundária ainda pode estar atuando na alteração da composição florística do fragmento estudado em toda sua extensão.

\section{AGRADECIMENTOS}

Ao biólogo Gilmar E. Valente e à professora Aristéa A. Azevedo, proprietários do sítio Palmital, por permitirem a execução deste estudo na área; à bióloga Renata B.F. Campos, pelo auxílio nos trabalhos de campo, e à CAPES e à FAPEMIG, pelo auxílio financeiro.

\section{REFERÊNCIAS BIBLIOGRÁFICAS}

ALMEIDA-JÚNIOR, J. S. Florística e fitossociologia de fragmentos da Floresta Estacional Semidecidual, Viçosa, Minas Gerais. 1999. 148 f. Dissertação (Mestrado em Ciência Florestal) - Universidade Federal de Viçosa, Viçosa, 1999. 
ALMEIDA, D. S; SOUZA, A. L. Florística e estrutura de um fragmento de Floresta Atlântica no município de Juiz de Fora, Minas Gerais. Revista Árvore, v. 21, p. 221-230, 1997.

BROWER, J. E.; ZAR, J. H. Field and laboratory methods for general ecology. Dubuque: Wm.CBronw, 1984. $226 \mathrm{p}$.

CARVALHO, D. A. et al. Florística e estrutura da vegetação arbórea de um fragmento de floresta semidecidual às margens do reservatório da usina hidrelétrica Dona Rita, Itambé do Mato Dentro, MG. Acta Botanica Brasilica, v. 14 , n. 1 , p. $37-55,2000$.

CASTRO, P. S. et al. Interceptação da chuva por mata natural secundária na região de Viçosa, MG. Revista Árvore, v. 7, p. 76-88, 1973.

CRONQUIST, A. The evolution and classification of flowering plants. New York: The New York Botanical Garden, 1988. 555 p.

DEPARTAMENTO NACIONAL DE METEOROLOGIA. Normais climatológicas (1961 - 1990). Brasília, 1992. $84 \mathrm{p}$.

FUNDAÇÃO CENTRO TECNOLÓGICO DE MINAS GERAIS-CETEC. Diagnóstico ambiental do Estado de Minas Gerais. Belo Horizonte: 1983. 158 p. (Série de Publicações Técnicas, 10).

GOLFARI, L. Zoneamento ecológico do Estado de Minas Gerais. Belo Horizonte: Centro de Pesquisa Florestal da Região do Cerrado, 1975. 65 p.

LEITÃO-FILHO, H. F. et al. Ecologia da Mata Atlântica em Cubatão. São Paulo: Editora da Unesp e Editora da Unicamp, 1993. $184 \mathrm{p}$.

MARANGON, L. C. Florística e fitossociologia de área de Floresta Estacional Semidecidual visando dinâmica de espécies florestais arbóreas no município de Viçosa, MG. 1999. 139 f. Tese (Doutorado em Ciências) - Universidade Federal de São Carlos, São Carlos, 1999.

MARISCAL-FLORES, E. J. Potencial produtivo e alternativas de manejo sustentável de um fragmento de Mata Atlântica secundária, Município de Viçosa, Minas Gerais. 1993. 165 f. Dissertação (Mestrado em Ciência Florestal) - Universidade Federal de Viçosa, Viçosa, 1993.

MEIRA-NETO, J. A. A. Estudos florísticos, estruturais e ambientais nos estratos arbóreo e herbáceo-arbustivo de uma floresta estacional semidecidual em Viçosa, MG. 1997. 154 f. Tese (Doutorado em Ciências Biológicas) Universidade Estadual de Campinas, Campinas, 1997.
MEIRA-NETO, J. A. A.; SILVA, A. F. Caracterização dos fragmentos florestais das áreas de influência e diretamente afetada da UHE de Pilar, Vale do rio Piranga, Zona da Mata de Minas Gerais. Viçosa: FUNARBE - Universidade Federal de Viçosa, 1995. 57 p.

MEIRA-NETO, J. A. A. et al. Estrutura de uma floresta estacional semidecidual submontana em área de influência da Usina Hidrelétrica de Pilar, Ponte Nova, Zona da Mata de Minas Gerais. Revista Árvore, v. 21, p. 213-219, 1997.

OLIVEIRA-FILHO, A. T.; MACHADO, J. N. M. Composição florística de uma floresta semidecídua montana, na serra de São José, Tiradentes, Minas Gerais. Acta Botanica Brasilica, v. 7, n. 2, p. 71-88, 1993.

OLIVEIRA-FILHO, A. T.; SCOLFORO, J. R. S.; MELLO, J. M. Composição florística e estrutura comunitária de um remanescente de floresta semidecídua montana em Lavras, MG. Revista Brasileira de Botânica, v. 17, n. 2, p. 167182, 1994.

PAULA, A. Alterações florísticas e fitossociológicas da vegetação arbórea numa Floresta Estacional Semidecidual em Viçosa, MG. 1999. 87 f. Dissertação (Mestrado em Botânica) - Universidade Federal de Viçosa, Viçosa, 1999.

ROYAL BOTANIC GARDENS. Index Kewensis on compact disc - Manual. Oxford: Oxford University Press, 1993. $67 \mathrm{p}$.

SENRA, L. C. Composição florística e estrutura fitossociológica de um fragmento florestal da fazenda Rancho Fundo, na Zona da Mata, Viçosa, Minas Gerais. 2000. 66 f. Dissertação (Mestrado em Botânica) Universidade Federal de Viçosa, Viçosa, 2000.

SHEPHERD, G. J. Fitopac 1, manual do usuário. Campinas, SP: UNICAMP, 1996. 96 p.

SILVA, A. F.; FONTES, N. R. L.; LEITÃO-FILHO, H. F. Composição florística e estrutura horizontal do estrato arbóreo de um trecho da Mata da Biologia da Universidade Federal de Viçosa - Zona da Mata de Minas Gerais. Revista Árvore, v. 24, p. 397-405, 2000.

SNEATH, P. H.; SOKAL, R. R. Numerical taxonomy. San Francisco: W.H. Freeman and Company, 1973. 573 p.

SOARES-JÚNIOR, F. J. Composição florística e estrutura de um fragmento de Floresta Estacional Semidecidual na fazenda Tico-Tico, Viçosa, Minas Gerais. 2000. $68 \mathrm{f}$. Dissertação (Mestrado em Botânica) - Universidade Federal de Viçosa, Viçosa, 2000.

TABARELLI, M.; MANTOVANI, W. A regeneração de uma floresta tropical montana após corte e queima (São Paulo Brasil). Revista Brasileira de Biologia, v. 59, n. 2, p. 239251, 1999.

R. Árvore, Viçosa-MG, v.27, n.6, p.821-830, 2003 
VALVERDE, O. Estudo regional da Zona da Mata de Minas Gerais. Revista Brasileira de Geografia, v. 20, n. 1, p. 1-82, 1958.

VELOSO, H. P.; RANGEL FILHO, A. L. R.; LIMA, J. C. A. Classificação da vegetação brasileira, adaptada a um sistema universal. Rio de Janeiro: IBGE, 1991.

$123 \mathrm{p}$.
VIANELLO, R. L.; ALVES, A. R. Meteorologia básica e aplicações. Viçosa: Universidade Federal de Viçosa, 1991. $448 \mathrm{p}$.

WERNECK, M. S. et al. Florística e estrutura de três trechos de uma floresta semidecidual na Estação Ecológica do Tripuí, Ouro Preto, MG. Revista Brasileira de Botânica, v. 23, n. 1, p. 97-106, 2000. 